\title{
Revisión sistemática en revistas científicas de las publicaciones colombianas con uso de citometría de flujo
}

\begin{abstract}
Nicolás A. Giraldo, John M. González
Grupo de Ciencias Básicas Médicas, Facultad de Medicina, Universidad de los Andes, Bogotá, D.C., Colombia

Objetivo. Se realizó una búsqueda sistemática de la literatura científica con el objetivo de conocer el número de artículos colombianos publicados con el uso de citometría de flujo, sus aplicaciones y el aporte a la literatura mundial sobre el tema.

Método. Se utilizaron las bases de datos electrónicas PubMed, LILACS e ISI Web of Knowledge, y se buscaron los términos "flow cytometry" and "Colombia" o "citometría de flujo" y "Colombia". Se incluyeron trabajos de investigación experimentales que usaron la citometría de flujo y cuyo primero o último autor pertenecieran a grupos de investigación colombianos.

Resultados. Se encontraron 74 artículos que cumplían los criterios de inclusión, publicados desde agosto de 1997 hasta marzo de 2010. La gran mayoría de los trabajos pertenecía al área biomédica y en $54 \%$ se aplicó al estudio de enfermedades infecciosas. El número de publicaciones por año aumentó desde 1997 hasta 2004, cuando se presentó un pico de 11 artículos. En total, se encontraron 27 grupos de investigación, pertenecientes a 13 instituciones. El 39,2\% (n=29) de los trabajos fueron publicados por grupos de investigación de la Universidad de Antioquia. El tipo de aplicación de la citometría de flujo más usada fue el análisis de inmunofenotipo $(58,1 \%)$.

Conclusiones. Para el periodo comprendido entre agosto de 1997 y marzo de 2010, PubMed reportó 76.312 publicaciones relacionadas con la citometría de flujo. Por consiguiente, el aporte de las publicaciones colombianas a la literatura mundial es tan sólo de 0,096\%.
\end{abstract}

Palabras clave: citometría de flujo, Colombia

Systematic revision in scientific journals of Colombian publications with the use of flow cytometry

Objective: In this work, a systematic review of the scientific literature was carried out in order to determine the number of Colombian scientific publications involving the use of flow cytometry. Special focus was made on the use of flow cytometry applications in these publications, and the contribution of Colombian publications to world scientific knowledge.

Methods: The databases used to carry out the searches were PubMed, LILACS and ISI Web of Knowledge, and the searched terms were "flow cytometry" and "Colombia" or "citometría de flujo" and "Colombia". Experimental papers that used flow cytometry where included in which the first or last author belonged to a Colombian research group.

Results: A total of 74 articles published from August, 1997, to March, 2010, were compiled. Most of the papers were from the biomedical field and $54 \%$ of them were related to infectious diseases. The number of publications per year increased progressively from 1997 until 2004 where a peak of 11 papers was registered. These publications were from 27 research groups that belong to 13 different institutions. Groups from Universidad de Antioquia contributed with $39.2 \%(n=29)$ of the published papers. The application of flow cytometry more referred was immunophenotype analysis with $58.1 \%$.

Conclusions: Until May, 2010, PubMed reported 76,312 publications related to flow cytometry; therefore, Colombian publications contributed $0.096 \%$ of the world publications in this topic.

Key words: flow cytometry, Colombia

La investigación sobre la inmunidad celular, a diferencia de la larga historia en el desarrollo de la evaluación de la inmunidad humoral, ha prosperado

Correspondencia:

John M. González, Facultad de Medicina, Universidad de los Andes, Carrera 1 № 18A-10 edificio Q, piso 8, Bogotá, D.C., Colombia.

Teléfono: 339 4949, extensión 3718: fax: 3324281

johgonza@uniandes.edu.co en los últimos 40 años gracias a la aparición de técnicas como la citometría de flujo.

El uso de la separación de células identificadas por medio de marcadores específicos, se publicó en 1969 y fue una de las primeras aplicaciones de esta técnica, en la que se combina la suspensión de células en un flujo con la detección de sus características morfológicas y la presencia de 
fluorescencia (1). Dicha aplicación dio el origen al nombre en inglés de los equipos de citometría, el cual se ha popularizado en todo el mundo con la sigla FACs (fluorescence activated cell sorting) (2). Los nuevos equipos con la utilización de uno a tres rayos láser, la detección de varias longitudes de onda emisoras y la marcación de los anticuerpos monoclonales con diferentes fluorocromos, permiten el análisis de múltiples parámetros en una sola célula, aportando al estudio de patrones de expresión celular en condiciones normales y patológicas (3). La aplicación inicial de la citometría de flujo en la práctica clínica fue el conteo de linfocitos T en la infección por el virus de la inmunodeficiencia humana (4).

El auge del uso de esta tecnología se refleja en el número de publicaciones para marzo de 2010 en la página de la U. S. National Library of Medicine (http://www.ncbi.nlm.nih.gov/pubmed/).Se encontraron 107.529 artículos al usar las palabras clave "flow" y "cytometry", y la primera publicación se hizo en 1973 (5).

En el laboratorio y en la práctica clínica, sus aplicaciones se relacionan con la identificación de moléculas celulares (inmunofenotipo), pruebas funcionales en leucocitos, glóbulos rojos y plaquetas; pruebas con ácidos nucleicos, que incluyen apoptosis, proliferación, ciclo celular y contenido de ADN, además de la detección de factores solubles y determinación de células específicas de antígeno (3,6-8). En la actualidad, la citometría es una pieza clave en el diagnóstico de las inmunodeficiencias primarias: no sólo la medición de la cantidad de células, sino también, pruebas funcionales y la presencia o ausencia de proteínas asociadas a las deficiencias (9).

El objetivo de este trabajo fue hacer una búsqueda sistemática del aporte de las publicaciones colombianas sobre el uso de citometría de flujo, en la literatura mundial indexada en inglés y español.

\section{Metodología}

Con el objetivo de conocer el número de artículos publicados en revistas indexadas y realizados por grupos de investigación de Colombia que usan la citometría de flujo, se hizo una búsqueda sistemática en las bases de datos electrónicas PubMed, LILACS e ISI Web of Knowledge. La fecha final del análisis fue marzo de 2010 y la búsqueda incluyó artículos publicados en español o en inglés. Para la estrategia de búsqueda, se usaron los términos en inglés definidos en MeSH (medical subject headings): "flow cytometry" en combinación con "Colombia"; no se utilizó el término "cytometry", pues se asignaba esta terminología a otro tipo de técnicas. Para la búsqueda en español, se usaron los siguientes términos definidos en DeCS (descriptores en ciencias de la salud): "citometría de flujo" junto con "Colombia".

Los criterios de inclusión fueron los siguientes:

a) uso de citometría de flujo en la metodología del trabajo;

b) primero o último autor perteneciente a grupos de investigación establecidos en Colombia, o un número igual o mayor de tres autores que cumplieran este criterio, $y$

c) trabajos de investigación experimentales.

De cada artículo se extrajeron los siguientes datos: autores, título del artículo, año de publicación, grupo de investigación, idioma, palabras clave y tipo de aplicaciones en la que se utilizó citometría de flujo. Cuando los grupos de investigación a los que pertenecían el primero y el último autor diferían, se tomó en cuenta el último únicamente.

Los temas de los estudios se categorizaron de la siguiente manera:

a) tipo de célula por expresión de moléculas tanto de superficie como intracelulares (inmunofenotipo), incluyendo fenotipo de leucemias y linfomas;

b) pruebas funcionales en leucocitos;

c) apoptosis;

d) proliferación, ciclo celular y contenido de ácidos nucleicos;

e) detección de productos solubles de leucocitos;

f) detección de linfocitos $T$ y $B$ específicos de antígeno;

g) células madre hematopoyéticas;

h) detección de anticuerpos y complejos antígenoanticuerpo;

i) plaquetas y glóbulos rojos, y

j) pruebas de histocompatibilidad.

Un mismo estudio podía ser categorizado en varias aplicaciones $(3,6-8)$. Los datos se muestran como porcentajes de publicaciones por año, por instituciones y por tema.

\section{Resultados y discusión}

La búsqueda en PubMed arrojó 68 artículos, de los cuales, se excluyeron seis por no cumplir 
los criterios de inclusión referentes a grupos de investigación, y dos más porque su metodología no utilizaba la citometría de flujo. La búsqueda en LILACS arrojó cuatro artículos, de los cuales, uno se excluyó porque era un artículo de revisión. La base de datos ISI Web of Knowledge arrojó 21 artículos nuevos, de los cuales, se excluyeron doce por no cumplir los criterios de inclusión referentes a grupos de investigación.

En consecuencia, se analizaron 74 artículos publicados entre agosto de 1997 y marzo de 2010. En su mayoría, los trabajos de investigación se pueden clasificar como del área biomédica; el 54\% $(n=40)$ de los mismos trataba sobre enfermedades infecciosas; Mycobacterium tuberculosis y Leishmania spp. fueron los microorganismos más frecuentemente estudiados, con un total de ocho y siete publicaciones, respectivamente. Otros temas publicados fueron las enfermedades inflamatorias/ alérgicas $(n=13)$ y los trasplantes de órganos $(n=5)$. Cuatro artículos pertenecían al área de ciencias biológicas, incluidos tres en los cuales se determinó el contenido de ADN en Coffea arabica y en otro se hizo la misma cuantificación en camarón marino (Litopenaeus vannamei).

El primer artículo con uso de citometría de flujo fue publicado en agosto de 1997. Entre 1998 y 2001 hubo un número bajo de publicaciones (menos de cuatro por año) y, en el 2002, este número aumentó. Existe un pico de once publicaciones en 2004 (figura 1). El aumento de las publicaciones de 2002 en adelante, probablemente, se debió a que otros grupos de investigación diferentes a los de la Universidad Nacional de Colombia, sede Bogotá, y la Facultad de Medicina de la Universidad de Antioquia, empezaron a publicar sobre el tema a partir de este año, entre ellos la Pontificia
Universidad Javeriana y la Universidad del Cauca. Desde el 2004 hasta el 2009, excepto por el 2006, se publicaron entre ocho y nueve trabajos por año (figura 1).

En la distribución por instituciones y grupos, se encontraron 27 diferentes grupos de investigación pertenecientes a 13 instituciones. El 39,2\% ( $n=29)$ de los trabajos fueron publicados por grupos de investigación pertenecientes a la Universidad de Antioquia, entre ellos, el Grupo de Inmunología Celular e Inmunogenética ( $n=12)$ y el Grupo de Inmunovirología $(n=4)$. La segunda institución con mayor número de publicaciones fue la Pontificia Universidad Javeriana de Bogotá, con 18,9\% $(n=14)$ de las publicaciones, donde se destacaron el Instituto de Genética Humana $(n=5)$ y el Grupo de Inmunobiología y Biología Celular $(n=4)$. La tercera institución fue la Universidad Nacional de Colombia con sede en Bogotá, incluido el Instituto de Inmunología del Hospital San Juan de Dios con $10,8 \%(n=8)$, y la cuarta fue la Fundación Instituto de Inmunología de Colombia (FIDIC), con 8,1\% ( $n=6)$. El restante $23,0 \%(n=17)$ fue publicado por otras instituciones, que incluían: la Universidad del Cauca, el Centro Nacional de Investigaciones del Café, la Corporación para Investigaciones Biológicas (CIB), el Instituto Nacional de Cancerología, el Centro Internacional de Entrenamiento e Investigaciones Médicas (CIDEIM), la Universidad del Valle, el Centro de Investigaciones de la Acuicultura en Colombia, el Banco de Células Stem de Colombia y la Universidad de Caldas; esta distribución se muestra en la figura 2.

Al categorizar cada estudio según el tipo de aplicaciones en la que se utilizó la citometría de flujo, se encontró que el análisis de inmunofenotipo fue la metodología más frecuente, usada en 43

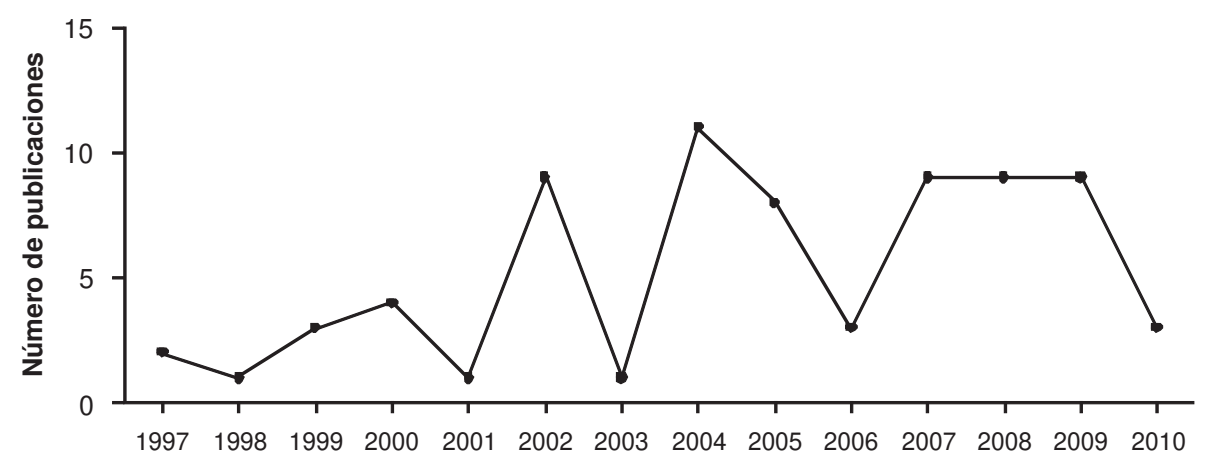

Año

Figura 1. Número de trabajos publicados entre 1997 y 2010 en revistas indexadas, que usaron citometría de flujo en su metodología y cuyo grupo de investigación estaba establecido en Colombia. 


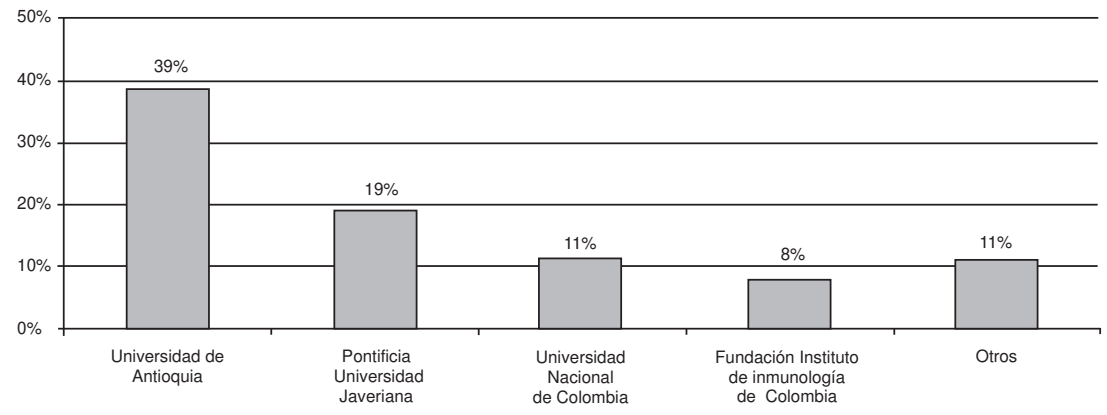

Figura 2. Porcentaje de artículos publicados por cada institución sobre el total de las publicaciones encontradas.

Cuadro 1. Número de publicaciones según aplicaciones. Se muestra el número de trabajos de investigación categorizados según las aplicaciones de la citometría de flujo.

\begin{tabular}{lc}
\hline Temas & $\mathbf{n}$ \\
\hline Identificación de marcadores (inmunofenotipo) & 43 \\
Pruebas funcionales en leucocitos & 21 \\
Apoptosis & 10 \\
Proliferación, ciclo celular y contenido de ácidos nucleicos & 9 \\
Detección de productos solubles de leucocitos & 7 \\
Detección de linfocitos T y B específicos de antígeno & 6 \\
Células madre hematopoyéticas & 2 \\
Detección de anticuerpos y complejos inmunitarios & 2 \\
Plaquetas y glóbulos rojos & 2 \\
Pruebas de histocompatibilidad & 2 \\
Otros & 4
\end{tabular}

trabajos de investigación (58,1\%), incluyendo un trabajo en el que se estudiaba el fenotipo de leucemias y linfomas. Para ello, las moléculas de superficie analizadas con mayor frecuencia fueron CD3, CD4, CD8, CD11C, CD14, CD16, CD20, CD21, CD25, CD45RA, CD56, CD69, CD86, CD123, HLA-DR, IgG1 e lgG2a.

La segunda aplicación más utilizada fueron las pruebas funcionales en leucocitos, $28,4 \%(n=21)$, que incluyó la medición de citocinas intracelulares (más frecuentemente IL-1a, IL-12, FNTa, IL-4 e IFN- $\gamma$ ) y fagocitosis/endocitosis de bacterias (principalmente, M. tuberculosis).

Otras aplicaciones frecuentemente utilizadas fueron el estudio de la apoptosis, 13,5\% $(n=10)$, y la proliferación, el ciclo celular y el contenido de ADN, $12,2 \%(n=9)$.

El número de publicaciones para cada tipo de aplicación en la que se utilizó citometría de flujo, se presenta en la tabla 1. La proporción entre las metodologías usadas permaneció estable durante el periodo evaluado de 1997 a 2010. Teniendo en cuenta las 76.312 publicaciones sobre citometría de flujo que aparecen en PubMed para la fecha del análisis, en el periodo comprendido entre agosto de 1997 y marzo de 2010, Colombia aportó tan sólo el 0,096\% de las publicaciones mundiales en el tema.

\section{Referencias}

1. Tung JW, Heydari K, Tirouvanziam R, Sahaf B, Parks DR, Herzenberg LA, et al. Modern flow cytometry: a practical approach. Clin Lab Med. 2007;27:453-68.

2. Hulett HR, Bonner WA, Barrett J, Herzenberg LA. Cell sorting: automated separation of mammalian cells as a function of intracellular fluorescence. Science. 1969;166:747-9.

3. Scheffold A, Kern F. Recent developments in flow cytometry. J Clin Immunol. 2000;20:400-7.

4. Prince HE, Arens L, Kleinman SH. CD4 and CD8 subsets defined by dual-color cytofluorometry which distinguish symptomatic from asymptomatic blood donors seropositive for human immunodeficiency virus. Diagn Clin Immunol. 1987;5:188-93. 
5. Fulwyler MJ. Status quo in flow-through cytometry. J Histochem Cytochem. 1974;22:605-6.

6. Wright JF, Lazarus AH, Freedman J. Applications of flow cytometry in the analysis of blood leukocytes. Transfus Sci. 1995;16:333-41.

7. Orfao A, Ruiz-Arguelles A, Lacombe F, Ault $K$, Basso G, Danova M. Flow cytometry: its applications in hematology. Haematologica. 1995;80:69-81.
8. Brown M, Wittwer C. Flow cytometry: principles and clinical applications in hematology. Clin Chem. 2000;46:1221-9.

9. Oliveira JB, Notarangelo LD, Fleisher TA. Applications of flow cytometry for the study of primary immune deficiencies. Curr Opin Allergy Clin Immunol. 2008;8:499-509. 